\title{
Comparison of diagnostic methods in Cutaneous Leishmaniasis in Iraq
}

\author{
Abdulsadah A. Rahi ${ }^{1}$, Sundus Nsaif ${ }^{2}$, Jaafar J. Hassoni ${ }^{3}$, Magda A. Ali ${ }^{4}$, Hassan A. Hamza \\ ${ }^{1}$ College of Science/Wasit University, Iraq \\ ${ }^{2}$ College of Medicine/Kufa University, Iraq \\ ${ }^{3}$ College of Medicine/Wasit University, Iraq \\ ${ }^{4}$ College of Medicine/Wasit University, Iraq, TUMS-IC \\ ${ }^{5} \mathrm{Al}-$ Sadir Hospital/Ministry of Health, Iraq
}

\section{Email address:}

abdulsadah1966@yahoo.com (A. A. Rahi)

\section{To cite this article}

Abdulsadah A. Rahi, Sundus Nsaif, Jaafar J. Hassoni, Magda A. Ali, Hassan A. Hamza. Comparison of Diagnostic Methods inCutaneous Leishmaniasis in Iraq, American Journal of BioScience. Vol. 1, No. 1, 2013, pp. 1-5. doi: 10.11648/j.ajbio.20130101.11

\begin{abstract}
Sixty -four specimens collected passively from patients with cutaneous ulcers suggestive of leishmaniasis attending hospital and health centres in all parts of Iraq for diagnosis were included in the study. The present study was conducted from June to December 2012 in Al-Karamah Teaching Hospital in Wasit Province, Iraq. Dermal scrapings were analysed both by parasitological (examination of Giemsa-stained smears and in vitro cultivation) methods and by a genusspecific PCR detection. Microscopy revealed amastigotes in 38 samples (59.4\%) and in vitro cultivation gave positive results in 19 cases (29.7\%), whereas PCR detected Leishmania in 44 samples $(68.75 \%)$. There are two forms of Cutaneous leishmaniasis (CL) in Iraq are caused by two species of Leishmania. Of 44 well-documented cases in our study ; 28 $(63.6 \%)$ were caused byLeishmania major and $16(36.4 \%)$ by Leishmania tropica.
\end{abstract}

Keywords: Cutaneous Leishmaniasis, PCR, Diagnosis

\section{Introduction}

Molecular epidemiology is a fairly new branch of science that has emerged in the last 20 years in parallel with the development of molecular biology ${ }^{(1)}$. Leishmaniae are sand fly-borne parasites that appear as intracellular amastigotes within the macrophage phagosomes of the mammalian host, and as extracellular flagellated promastigotes in the gut of the sand fly or in vitro culture. Many species of sand flies are vectors of leishmaniasis. The most common are the Phlebotomus species in the eastern hemisphere, or Old World, and the Lutzomyia species in the western hemisphere, or New World. Generally, leishmaniasis is a zoonosis with humans acting as incidental hosts and other mammals (especially rodents and dogs) acting as reservoir hosts. There are, however, exceptions to this rule: Both Leishmania tropica and Leishmania donovani infection can be transmitted anthroponotically ${ }^{(2)}$.

Generally, leishmaniasis is divided into three clinical syndromes: cutaneous, mucosal, and visceral infection. Cutaneous leishmaniasis is localized to skin. Mucosal leishmaniasis occurs mainly as a late complication in new- world CL and is associated particularly with infection by Leishmania (Viannia) species. Visceral leishmaniasis is caused predominately by L. donovani and Leishmania infantum-chagasi, though in the Arabian Gulf War, L. tropica caused a mild form of visceral disease referred to as viscerotropic leishmaniasis ${ }^{(3)}$.

Cutaneous leishmaniasis (CL) caused by L. tropica and L. major are indistinguishable on clinical bases as both erupt in the same way, the size of the lesion ranging from a few millimeters to 4 centimeter or more. The site and number of lesions(s) are an indication of the type of CL. Leishmania major usually presents as multiple lesions $(\geq 3)$ and L. tropica is more often on the nose ${ }^{(4,5)}$. Cutaneous leishmaniasis is widespread throughout the country, except for the three provinces in the northeast, bordering Turkey and Iran, where cases are rare. It seems that the majority of CL cases reported in Iraq are caused by L.major (ZCL) ${ }^{(6)}$. Two epidemic outbreaks of CL have been reported in Diwania Province in 2008 with about 300 cases and in Baghdad/ Rahmania in 2009 with about 400 cases. These may have been caused by CL by L.tropica (ACL), a very old disease in Iraq, also called "Baghdad boil", which used to be com- 
mon until a few decades ago ${ }^{(7)}$.

Cutaneous leishmaniasis occurs either as a zoonotic or as an anthroponotic infection. The transmission of Leishmania major causing Old World cutaneous leishmaniasis in humans is a rural zoonotic disease with rodents serving as the reservoir. In zoonotic leishmaniasis, humans are only accidental hosts and usually not directly involved in the transmission cycle. New endemic foci of CL may occur, when environmental changes take place such as rise of population under poor housing conditions (slums) or specific forms of agriculture ${ }^{(8)}$.

The main insect vector for transmission of L. major is the sand fly species Phlebotomus papatasi ${ }^{(9)}$. In some urban centers of Middle East and Asia exist completely anthroponotic life cycles of the parasites, i.e. human beings are the main or only reservoir host. In such places cutaneous leishmaniasis caused by L. tropica can be highly endemic, but no animal reservoir is to be recognized. In Central and South Western Morocco, the transmission of L. tropica is anthroponotically as well. The parasite is mainly transmitted by sandflies of the species Phlebotomus sergenti ${ }^{(10)}$.

Diagnosis of leishmaniasis is based on clinical, parasitological, serological and molecular identification. Cutaneous leishmaniasis appear as papule or ulcer with crust center and raised edges ${ }^{(11)}$. The serological tests based on the presence of antibody in the blood in visceral leishmaniasis (VL) and cell mediated immunity in cutaneous and mucocutaneous leishmaniasis ${ }^{(12)}$ such as Leishmania skin test (Montenegro reaction), Indirect Fluorescent Antibody Test (IFAT), Direct Agglutination Test (DAT), and Enzyme Linked Immunosorbant assay (ELISA). The molecular tests are based on detection of nucleic acid such as Polymerase Chain Reaction (PCR) $)^{(13)}$. The aims of this study to evaluate the reappraisal of the diagnosis and epidemiology of CL in some parts of Iraq, by different parasitological, cultural, and molecular assay.

\section{Materials and Methods}

\subsection{Clinical Sample Collection}

Clinical samples consisted of all confirmed cases that visted to hospital and private clinics in some parts of Iraq from June 2012 till December 2012 with the symptoms of Cutaneous leishmaniasis and presence of amastigotes in Giemsa-stained smears. The demographic features including patient's sex, age, clinical signs including number of scars and its location in the body, month of diseases occurrence, incidence rate, and geographical regions were recorded.

Skin biopsies of 5 to $10 \mathrm{~mm}$ in diameter were taken under sterile conditions from the border of the ulcer and divided into three parts, The first part of the sample was smeared onto a glass slide, fixed with methanol, stained with Giemsa and examined by microscopy. A second part was inoculated on Novy-MacNeal-Nicolle (NNN) medium (14). The cultures were incubated at $24{ }^{\circ} \mathrm{C}$ and observed every week for 1 month. The third part for PCR was placed directly in lysis buffer (10 mM Tris-HCI, 10 mM EDTA).

\subsection{Microscopical Examination}

Small quantities of tissue obtained by skin scrapings were smeared on glass slides, air dried and fixed with methanol for a few seconds. Giemsa stain was filtered and diluted 1:20 with phosphate buffer (pH 7.2). After 20 minutes of staining the slides were washed with tap water and air dried. The stained smears were examined under the microscope with a $40 \mathrm{x}$ lens and with a $100 \mathrm{x}$ oil immersion lens. If at least one intra- or extra-cellular amastigote with a distinctive kinetoplast was found the smear was declared positive. When no amastigotes were seen after 15 minutes of inspection, the smear was declared negative. Many of the patient smears were double checked, the observations were in concordance.

\subsection{Culture}

The lesions and the adjacent normal-looking skin around them were cleaned, sterilized with $70 \%$ ethanol, and allowed to dry. Similar to the preparation of the slide smears, a small amount of the scraped tissue was inoculated on the liquid phase of Novy-McNeal-Nicolle (NNN) medium $(10 \%$ of rabbit blood). The cultures were incubated at $25^{\circ} \mathrm{C}$ and examined for parasite growth by the inverted microscope and also light microscope every 4 days until promastigotes were seen or up to one month before being discarded as negative. The cultures were made at least in duplicates for each case ${ }^{(15)}$.

\subsection{DNA Extraction}

Each fresh or dried smear was scraped off the slide with a sterile scalpel and the scrapings were added to $200 \mu \mathrm{l}$ lysis buffer [50 mM Tris- $\mathrm{HCl}$ (pH 7.6), 1 mM EDTA, 1\% $(\mathrm{v} / \mathrm{v})$ Tween 20] containing $8.5 \mu \mathrm{l}$ of a proteinase $\mathrm{K}$ solution $(19 \mu \mathrm{g} / \mathrm{ml})$, in a $1.5 \mathrm{ml}$ tube (11). The tube was incubated for $2 \mathrm{~h}$ at $56^{\circ} \mathrm{C}$ before $200 \mu \mathrm{l}$ of a phenol: chloroform isoamyl-alcohol mixture (25:24:1, by volume) was added. After being shaken vigorously, the tube was centrifuged at $6000 \mathrm{~g}$ for $10 \mathrm{~min}$ and then the DNA in the supernatant solution was precipitated with $400 \mu \mathrm{l}$ cold absolute ethanol, resuspended in $50 \mu \mathrm{l}$ double distilled water and then stored at $-20^{\circ} \mathrm{C}$, until it could be tested for leishmania kDNA $^{(16)}$.

\subsection{PCR}

PCR assay was performed according to the manufacturer's protocol (Sinagen, Iran) with the final volume of $25 \mu \mathrm{L}$ of each PCR reaction. PCR amplification was carried out in a DNA Thermal Cycler ( Master cycler gradient, San Leonardo, Canada) based on the following conditions: initial denaturation $\left(95^{\circ} \mathrm{C}, 3 \mathrm{~min} ; 63^{\circ} \mathrm{C}, 30 \mathrm{~s} ; 72^{\circ} \mathrm{C}, 60 \mathrm{~s}\right) 1$ cycle followed by 35 cycles including denaturation $\left(93^{\circ} \mathrm{C}, 20 \mathrm{~s}\right)$, annealing $\left(63^{\circ} \mathrm{C}, 20 \mathrm{~s}\right)$ and extension $\left(72^{\circ} \mathrm{C}, 40 \mathrm{~s}\right)$. Finally, $10 \mu \mathrm{L}$ of amplified samples without adding loading buffer 
were loaded in a $2 \%$ agarose gel containing $0.5 \mathrm{mg} / \mathrm{mL}$ ethidium bromide in electrophoresis and the products were visualized by Ultraviolet (UV) transillumination.

\subsection{Statistical Analysis}

The suitable statistical methods were used in order to analyze and assess the results. These were used to accept or reject the statistical hypotheses. All the statistical analysis were done by using Pentium-4 computer through the Minitab program and Excel application ${ }^{(17)}$.

\section{Results}

Table 1. show the prevalence of positive cases of CL by using different diagnostic methods. The highest infection (68.75\%) appeared by using PCR while the lowest infection $(29.7 \%)$ appeared by culture on NNN media.

Table 1. Distribution of positive cases of CL by different diagnostic methods.

\begin{tabular}{llcl}
\hline Test & \multicolumn{2}{l}{ Positive (\%)Negative (\%) } & Total \\
\hline Direct smear by Giemsa & $38(59.4)$ & $26(40.6)$ & 64 \\
Culture on NNN media & $19(29.7)$ & $45(70.3)$ & 64 \\
PCR & $44(68.75)$ & $20(31.25)$ & 64 \\
\hline
\end{tabular}

Table2. represents the diagnosis of two species of Leishmania by using PCR method. The present studywas revealed that the highest infection $(63.6 \%)$ caused by L.major than L.tropica (36.4\%).

Table 2. PCR reaction of CL samples.

\begin{tabular}{lcc}
\hline PCR reaction & L.major(\%) & L.tropica (\%) \\
\hline Positive & $28(63.6)$ & $16(36.4)$ \\
Negative & $16(36.4)$ & $28(63.6)$ \\
\hline
\end{tabular}

The comparison of the clinical features of CL cases was shown in table 3 . Our study appeared that median duration of lesions was under 2 month ( 64.3\%) in L.major cases and ( $68.75 \%$ ) in L.tropica. Results of this study showed that most ulcers $(64.3 \%)$ were in face and thirteen patients $(46.4 \%)$ presented with single lesion.

Table 3. Comparison of the clinical features of CL cases.

\begin{tabular}{lll}
\hline Clinical features & L.major\% & L.tropica\% \\
\hline Duration (months) & & \\
$<2$ & 1864.3 & 1168.75 \\
$2-4$ & 828.6 & 425.0 \\
$>4$ & 27.1 & 16.25 \\
Total & 28100 & 16100 \\
Location & & \\
Forehead & 1633.3 & 1442.4 \\
Eye & 816.6 & 618.2 \\
Nose & 612.5 & 412.1 \\
Ear & 36.25 & 26.1 \\
Arm & 816.6 & 412.1 \\
Leg & 36.25 & 13.0 \\
\hline
\end{tabular}

\begin{tabular}{lll}
\hline Clinical features & L.major\% & L.tropica\% \\
\hline Neck & 24.2 & 13.0 \\
Abdomen & 24.2 & 13.0 \\
Total & 4899.9 & 3399.9 \\
Number of Lesions & & \\
1 & 1346.4 & 850.0 \\
2 & 1139.3 & 531.25 \\
$\geq 3$ & 414.3 & 318.75 \\
Total & 28100 & 16100 \\
\hline
\end{tabular}

Regarding gender differences, in the study areas, CL have been reported more frequently in males $(54.5 \%)$ than females $(45.5 \%)$ and high prevalence $(22.7 \%)$ in age group under six years old ( Table 4).

Table 4. Distribution of positive cases of CL in relation to the age and gender.

\begin{tabular}{llll}
\hline Age group (year) & Male (\%) & Female (\%) & Total (\%) \\
\hline $0-6$ & $10(22.7)$ & $9(20.5)$ & $19(43.2)$ \\
$7-12$ & $8(18.2)$ & $5(11.4)$ & $13(29.6)$ \\
$13-18$ & $4(9.1)$ & $4(9.1)$ & $8(18.2)$ \\
$\geq 19$ & $2(4.5)$ & $2(4.5)$ & $4(9.0)$ \\
Total & $24(54.5)$ & $20(45.5)$ & $44(100)$ \\
\hline
\end{tabular}

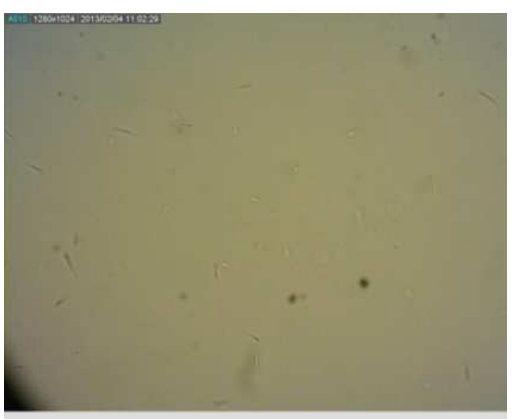

Figure 1. Promastigote forms ofLeishmania spp.

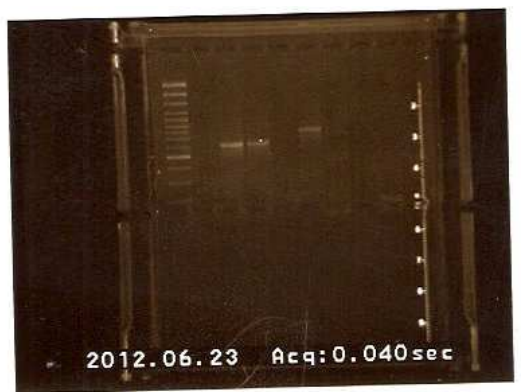

Figure 2. Electrophoretic patterns of PCR products obtained from crude parasite genomic DNAs for Leishmania species detection M; marker, 1; L.tropica (500 bp), 2 ; L.major (600 bp).

\section{Discussion}

A round shape Leishmania parasite without flagellum was grown in culture media with biphasic NNN medium as demonstrated by light microscopy examination. The change of promastigotes to amastigotes did take place completely in culture with NNN medium. These finding again emphas- 
ize that optimal condition for propagation of axenic amastigotes vary and have to be determined for each Leishmania species isolates. DNA isolated from promastigote forms obtained from in vitro culture of Leishmania allowed for optimization of PCR reaction ${ }^{(18)}$.

The diagnosis of CL classically relies on microscopic examination and in vitro cultivation. These classical methods require the presence of a relatively high number of viable or morphologically intact parasites; this may pose a problem particularly in the chronic phase of CL where parasite levels in skin lesions are very low. In contrast, the molecular approach is both sensitive and specific ${ }^{(19)}$. In this study we set up a well documented, genus-specific PCR to detect Leishmania species in clinical cutaneous samples and compared this method with classical methods. The PCR-based checking of Giemsa-stained smears appears to be reasonably, sensitive and specific in revealing the presence of Leishmania parasites in such chronic lesions. PCR can clearly help improve the diagnosis ofCL in these difficult cases.

Sixty-four patients with 81 skin lesions were enrolled in our study : 24 males (54.5\%) and 20 females (45.5\%). The highest infection $(68.75 \%)$ appeared by using PCR while the lowest infection $(29.7 \%)$ appeared by culture on NNN media .The high prevalence $(40.9 \%)$ in age group between (1 day -12 years). In Iraq, cutaneous leishmaniasis (Baghdad boil) caused by two species L. major zoonotic disease and L.tropica anthroponotic disease ${ }^{(20)}$. The present study revealed that prevalence of L. major (63.6\%) were higher than L.tropica $(36.4 \%)$ in the studied areas. Similar to the findings were recorded of some other studies ${ }^{(21,22)}$.

Most of the CL cases (68.75\% for L.tropica and $64.3 \%$ for L.major) were had duration of lesions under 2 years old and most affected part of the body was face $(64.3 \%)$ with single lesions(46.4\%). This can be due to the fact that some ulcers do not necessarily lead to the appearance of scars for several possible reasons, i.e. immune system interference or early healing of the ulcers, spontaneously or due to treatment. These results with agreements with other studies in Iraq ${ }^{(23)}$ and other countries ${ }^{(24-26)}$.

Regarding gender differences, in the study areas, CL have been reported more frequently in men $(54.5 \%)$ in comparison to women $(45.5 \%)$. The reason is that more men work or sleep in open areas and is also due to men's less covering than women and more exposure to the infected sand flies ${ }^{(27)}$. Some studies have hypothesized that the gender difference observed in some parasitic disease can be attributed to hormonal effects. However, controversy still exists regarding the role of sex hormones in the cellular immune response ${ }^{(28,29)}$. Although it is believed that sex hormones may influence the establishment and the course of parasitic diseases, behavioral factors, making male individuals more likely to be exposed to vectors in fields and other transmission environments, are probably equally or more important ${ }^{(30,31)}$. On the contrary of other studies that found the higher incidence of infection among females than males ${ }^{(32-34)}$.
The majority of the CL cases (72.8\%) in age groups under 12 years old, which may be due to several factors, such as children's outdoor activities and sleeping outdoors, which increases exposure to sand fly bites during their active hours. The same result has been reported by some other researchers ${ }^{(25,32-35)}$.

\section{Conclusions}

Characterization of Leishmania isolates collected from different parts of Iraq showed that L.major and L.tropica are the agents of CL . Moreover, this study revealed that PCR is a reliable method for diagnosis and identification of Leishmania species and can applied in epidemiologic investigations in Iraq.

\section{References}

[1] Tazi, L., Kreiswirth, B., Carrière, C., Tibayrenc, M. Molecular epidemiology of Mycobacterium tuberculosis and its relevance to the surveillance and control of TB: an edebate. Infec. Gen. Evol., 2002; 2: 153-158.

[2] Dedet JP, Pratlong F. Leishmaniasis. In: Cook GC, Zumla AI, eds. Manson's Tropical Diseases. 21st ed. London, England: Elsevier Science Limited, 2003;1339-1364.

[3] Magill AJ, Grogl M, Gasser RA, Sun W, Oster CN. Visceral infection caused by Leishmania tropica in veterans of Operation Desert Storm. N Engl J Med., 1993; 328: 1383-1387.

[4] Klaus S, and Frankenburg S. Cutaneous Leishmaniasis in the Middle East, Clin. Dermatol.,1999; 17:137-141.

[5] Al-Jawabreh, A., Schnur, L. F., Nasereddin, A., Schwenkenbecher, J. M, Abdeen, Z., Barghuthy, F., Khanfar, H. Presber, $\mathrm{W}$ and Schoenian, G. The recent emergence of Leishmania tropica in Jericho (A'riha) and its environs, a classical focus of Leishmania major. Trop. Med. Int. Hlth., 2004; 9 (7): 812-816.

[6] WHO . Communicable Disease Working Group on Emergencies, HQ Division of Communicable Disease Control, EMRO, WHO Office, Baghdad. WHO Office, Baghdad. Communicable Disease Toolkit, Iraq Crisis.WHO, 2003; 39 $-44$.

[7] Abdulghani Mohamad AlSamarai, Hussein Saher AlObaidi. Cutaneous leishmaniasis in Iraq. J Infect Developing Countries , $2009 ; 3(2): 123-129$.

[8] Al-Jawabreh A, Diezmann S, Müller M, Wirth T, Schnur LF, Strelkova MV, Kovalenko DA, Razakov SA, Schwenkenbecher J, Kuhls K, and Schönian G. Identification of geographically distributed sub-populations of Leishmania (Leishmania) major by microsatellite analysis. BMC Evolutionary Biology. 2008; 8:183.

[9] El-Badry A, Al-Juhani A, El-Kheir I and Al-Zubiany S. Distribution of sand flies in El-Nekheil province, in AlMadinah Al-Munawwarah region, western of Saudi Arabia. Parasitol Res., 2008;103:151-156.

[10] Ramaoui K, Guernaoui S and Boumezzough A. Entomological and epidemiological study of a new focus of cutaneous leishmaniasis in Morocco. Parasitol Res., 2008; 103:859-863. 
[11] Levinson, W., Jawetz, E. Medical microbiology and immunology .7th ed. Lang Medical Book, McGrow. Hill. London. 2002;316-327.

[12] Herwaldt, B.L. Leishmaniasis. comprehensive review covering parasitology,epidemiology, clinical manifestations with variations, diagnosis and treatment. Lancet.1999; 354 : 11911199.

[13] Tavares, C. A. P., Fernandes, A. P., and Melo, M. N. Molecular diagnosis of leishmaniasis. Expert. Rev. Mol. Diagn., 2003; 3: 657-667.

[14] Singh S. New developments in diagnosis of leishmaniasis. Indian J. Med. Res. 2006; 123 (3): 311-30.

[15] Chomczynski, P. and Sacchi, N. "Single-step method of RNA isolation by acid guanidinium thiocyanate-phenolchloroform extraction: Twenty-something years on". Nature Prot. 2006; 1 (2): 581-585.

[16] Pratlong, F., Rioux, J.A., Marty, P., Faraut-Gambarelli, F., Dereure, J., Lanotte, G., Dedet, J.P. Isoenzymatic analysis of 712 strains of Leishmania infantum in the south of France and relationship of enzymatic polymorphism to clinical and epidemiological features. J. Clin. Microbiol., 2004; 42, 4077-4082.

[17] Al-Mashadani, K.A.K. and Abbodi E.H. Statistical hypothesis tests. Baghdad University, 2009.

[18] Pan AA, Duboise SM, Eperon S, Rivas L, Hodgkinson V, Traub-Cseko Y, Mc Mahon-Pratt D. Developmental life cycle of Leishmania cultivation and characterization of cultured exteracellular amastigotes. J Eurkaryot Microbiol., 1993; 40 (2): 213-23.

[19] Laskay, T., Miko, T.L., Negesse,Y., Solbach, W., Rollinghoff, M. \& Frommel, D. Detection of cutaneous Leishmania infection in parafin-embedded skin biopsies using the polymerase chain reaction. Transactions of the Royal Society of Tropical Medicine and Hygiene, 1995; 89: 273-275.

[20] Al-Jeboori T. I. and Evans D.A. Leishmania species in Iraq. Electrophoretic isoenzyme patterns II. Cutaneous Leishmaniasis. Trans. Roy. Soc. Trop. Med. Hyg., 1980 ; 74: 178184.

[21] Abdullah M. Qader, Mushriq K. Abood, and Tural Y. Bakir. Identification of Leishmania parasites in clinical samples obtained from Cutaneous Leishmaniasis patients using PCR technique in Iraq.Iraqi Journal of Science, 2009; 50(1):32 36.

[22] Alimoradi S.,Hajjaran H,Mohebali M, Mansouri F.Molecular Identification of Leishmania Species Isolated from Human Cutaneous Leishmaniasis by RAPD-PCR. Iranian J Publ Health, 2009; 38(2): 44-50.
[23] Khalifa E. Sharquie and Rafid A. Najim. Disseminated cutaneous leishmaniasis. Saudi Med J., 2004; 25 (7):951-954.

[24] Talari SA, Talaei R, Shajari G, Vakili Z, Taghaviardakani A. Childhood cutaneous leishmaniasis: report of 117 cases from Iran. Korean J Parasitol., 2006; 44: 355-60.

[25] Momeni AZ, Aminjavaheri M. Clinical picture of cutaneous leishmaniasis in Isfahan, Iran. Int J Dermatol., 1994; 33: $260-5$.

[26] Hojat A.N., Mehdi B., Mojtaba N., Mohamad M. Cutaneous Leishmaniasis in school children in border area at southwest of Iran. Sci Parasitol.,2012;13(4):153-158.

[27] Arroub H, Alaoui A, Habbari K. Etude Eco- Epidemiologique de la Leishmaniose Cutanée dans la Région de Foum Jamâa (Azilal, Maroc). Le Premier Colloque International sur les Changements Climatiques et Environnement. Faculté des Sciences, Rabat, Maroco, 2010.

[28] Bailey MS and Diana NJ. Cutaneous leishmaniasis. Clin Dermatol., 2007; 25: 203-211.

[29] Khan SJ, Muneeb S. Cutaneous leishmaniasis in Pakistan. Dermatol Online J., 2005; 11(4). [Online] Available from: http:// dermatology. cdlib.org/111/reviews/ leishmaniasis3/khan.html. [Accessed on 2007 Aug 24].

[30] Rastogi V, Nirwan PS. Cutaneous leishmaniasis: an emerging infection in a non-endemic area and a brief update. Indian J Med Microbiol., 2007; 25: 272-275.

[31] Stewart CC, Brieger WR. Community views on cutaneous leishmaniasis in Istalif, Afghanistan: implications for treatment and prevention. Int Quart Commun Health Education 2009; 29: 123-142.

[32] Fellah H, Rhajoui M, Ouahabi S, Belghiti D and Lyagoubi M.Occurrence of Human Cutaneous Leishmaniasis in Zouagha My Yacoub Province (Morocco). Int. J. Agri. Biol., 2007; 9 (1):197-198.

[33] Gurel MS, Ulukanligil M, Ozbilge H. Cutaneous leishmaniasis in Sanliurfa: epidemiologic and clinical features of the last four years (1997-2000). Int JDermatol., 2002; 41(1): 32-7.

[34] Aytekin S, Ertem M, Yağdıran O, Aytekin N. Clinicoepidemiologic study ofcutaneous leishmaniasis in Diyarbakir Turkey. Dermatol Online J., 2006; 12(3): 14.

[35] Yaghoobi-Ershadi MR, Akhavan AA, Zahraei-Ramazani AV, Abai MR, EbrahimiB, Vafaei-Nezhad R, Hanafi-Bojd AA, Jafari R. Epidemiological study in a new focus of cutaneous leishmaniasis in the Islamic Republic of Iran. East Mediterr Health J., 2003; 9(4):816-26. 